\title{
On the Caginalp for a Conserve Phase-Field with a Polynomial Potentiel of Order $2 p-1$
}

\author{
Narcisse Batangouna, Cyr Séraphin Ngamouyih Moussata, Urbain Cyriaque Mavoungou \\ Faculté des Sciences et Techniques Université Marien Ngouabi Brazzaville, République du Congo \\ Email: banarcissess@yahoo.fr, csmoussath@gmail.com,urbainmav@gmail.com
}

How to cite this paper: Batangouna, N., Moussata, C.S.N. and Mavoungou, U.C. (2020) On the Caginalp for a Conserve Phase-Field with a Polynomial Potentiel of Order $2 p-1$. Journal of Applied Mathematics and Physics, 8, 2744-2756.

https://doi.org/10.4236/jamp.2020.812203

Received: August 26, 2020

Accepted: December 4, 2020

Published: December 7, 2020

Copyright $\odot 2020$ by author(s) and Scientific Research Publishing Inc. This work is licensed under the Creative Commons Attribution International License (CC BY 4.0).

http://creativecommons.org/licenses/by/4.0/

\begin{abstract}
Our aim in this paper is to study on the Caginalp for a conserved phase-field with a polynomial potentiel of order $2 p-1$. In this part, one treats the conservative version of the problem of generalized phase field. We consider a regular potential, more precisely a polynomial term of the order $2 p-1$ with edge conditions of Dirichlet type. Existence and uniqueness are analyzed. More precisely, we precisely, we prove the existence and uniqueness of solutions.
\end{abstract}

\section{Keywords}

A Conserved Phase-Field, Polynomial Potentiel of Order $2 p-1$, Dirichlet Boundary Conditions, Maxwell-Cattaneo Law

\section{Introduction}

The Caginalp phase-field model

$$
\begin{gathered}
\frac{\partial u}{\partial t}-\Delta u+f(u)=\theta \\
\frac{\partial \theta}{\partial t}-\Delta \theta=-\frac{\partial u}{\partial t}
\end{gathered}
$$

proposed in [1], has been extensively studied (see, e.g., [2]-[7] and [8]). Here, $u$ denotes the order parameter and $\theta$ the (relative) temperature.

Furthermore, all physical constants have been set equal to one. This system models, e.g., melting-solidification phenomena in certain classes of materials.

The Caginalp system can be derived as follows. We first consider the (total) free energy

$$
\psi(u, \theta)=\int_{\Omega}\left(\frac{1}{2}|\nabla u|^{2}+f(u)-u \theta-\frac{1}{2} \theta^{2}\right) \mathrm{d} x,
$$


where $\Omega$ is the domain occupied by the materiel.

We then define the enthalpy $H$ as

$$
H=-\frac{\partial \psi}{\partial \theta}
$$

where $\partial$ denotes a variational derivative, which gives

$$
H=u+\theta .
$$

The governing equations for $u$ and $\theta$ are then given by (see [9])

$$
\begin{gathered}
\frac{\partial u}{\partial t}=-\frac{\partial \psi}{\partial u}, \\
\frac{\partial H}{\partial t}+\operatorname{div} q=0,
\end{gathered}
$$

where $q$ is the thermal flux vector. Assuming the classical Fourier Law

$$
q=-\nabla \theta,
$$

we find (1) and (2).

Now, a drawback of the Fourier Law is the so-called "paradox of heat conduction", namely, it predicts that thermal signals propagate with infinite speed, which, in particular, violates causality (see, e.g. [10] and [11]). One possible modification, in order to correct this unrealistic feature, is the Maxwell-Cattaneo Law.

$$
\left(1+\frac{\partial}{\partial t}\right) q=-\nabla \theta
$$

In that case, it follows from (7) that

$$
\left(1+\frac{\partial}{\partial t}\right) \frac{\partial H}{\partial t}-\Delta \theta=0
$$

hence,

$$
\frac{\partial^{2} \theta}{\partial t^{2}}+\frac{\partial \theta}{\partial t}-\Delta \theta=\frac{\partial^{2} u}{\partial t^{2}}+\frac{\partial u}{\partial t} .
$$

This model can also be derived by considering, as in [12] (see also [13]-[20]), the Caginalp phase-field model with the so-called Gurtin-Pipkin Law

$$
q(t)=-\int_{0}^{+\infty} k(s) \nabla \theta(t-s) \mathrm{d} s .
$$

for an exponentially decaying memory kernel $k$, namely,

$$
k(s)=\mathrm{e}^{-s} \text {. }
$$

Indeed, differentiating (11) with respect to $t$ and integrating by parts, we recover the Maxwell-Cattaneo Law (9).

Now, in view of the mathematical treatment of the problem, it is more convenient to introduce the new variable

$$
\alpha=\int_{0}^{t} \theta(s) \mathrm{d} s, \quad \theta=\frac{\partial \alpha}{\partial t},
$$

and we have, integrating (10) with respect to $s \in[0,1]$. 


$$
\frac{\partial^{2} \alpha}{\partial t^{2}}+\frac{\partial \alpha}{\partial t}-\Delta \alpha=-\frac{\partial u}{\partial t}
$$

where

$$
\alpha(t, x)=\int_{0}^{t} T(\tau, x) \mathrm{d} \tau+\alpha_{0}(x)
$$

is the conductive thermal displacement. Noting that $T=\frac{\partial \alpha}{\partial t}$, we finally deduce from (33) and (36)-(37) the following variant of the Caginalp phase-field system (see [17]):

$$
\begin{gathered}
\frac{\partial u}{\partial t}-\Delta u+f(u)=\frac{\partial \alpha}{\partial t} \\
\frac{\partial^{2} \alpha}{\partial t^{2}}+\frac{\partial \alpha}{\partial t}-\Delta \alpha=-\frac{\partial u}{\partial t}
\end{gathered}
$$

In this paper, we consider the following conserved phase-field model:

$$
\begin{gathered}
\frac{\partial u}{\partial t}+\Delta^{2} u-\Delta f(u)=-\Delta \frac{\partial \alpha}{\partial t} \\
\frac{\partial^{2} \alpha}{\partial t^{2}}+\frac{\partial \alpha}{\partial t}-\Delta \alpha=-\frac{\partial u}{\partial t}
\end{gathered}
$$

These equations are known as the conserved phase-field model (see [21]-[30]) based on type II heat conduction and with two temperatures (see [3] and [4]), conservative in the sense that, when endowed with Neumann boundary conditions, the spatial average of the order parameter is a conserved quantity. Indeed, in that case, integrating (18) over the spatial domain $\Omega$, we have the conservation of mass,

$$
\begin{gathered}
\langle u(t)\rangle=\langle u(0)\rangle, \quad t \geq 0 \\
\langle\cdot\rangle=\frac{1}{v o l \Omega} \int_{\Omega} \mathrm{d} x
\end{gathered}
$$

denotes the spatial average. Furthermore, integrating (19) over, we obtain

$$
\langle\alpha(t)\rangle=\langle\alpha(0)\rangle, \quad t \geq 0
$$

Our aim in this paper is to study the existence and uniqueness of solution of (17)-(39). We consider here only one type of boundary condition, namely, Dirichlet (see [31] [32] [33]).

\section{Setting of the Problem}

We consider the following initial and boundary value problem

$$
\begin{gathered}
\frac{\partial u}{\partial t}+\Delta^{2} u-\Delta f(u)=-\Delta \frac{\partial \alpha}{\partial t} \\
\frac{\partial^{2} \alpha}{\partial t^{2}}+\frac{\partial \alpha}{\partial t}-\Delta \alpha=-\frac{\partial u}{\partial t} \\
\left.u\right|_{\Gamma}=\left.\Delta u\right|_{\Gamma}=\left.\alpha\right|_{\Gamma}=0, \quad \text { on } \partial \Omega,
\end{gathered}
$$




$$
\left.u\right|_{t=0}=u_{0},\left.\alpha\right|_{t=0}=\alpha_{0}, \frac{\partial \alpha}{\partial t}=\alpha_{1}
$$

As far as the nonlinear term $\mathrm{f}$ is concerned, we assume that

$$
f \in C^{\infty}(R), f(0)=0
$$

Consider the following polynomial potential of order $2 p-1$

$$
f(s)=\sum_{i=1}^{2 p-1} a_{i} s^{i}, p \in N^{*}, p \geq 2 ; a_{2 p-1}=2 p b_{2 p} \geq 0
$$

The function $\mathrm{f}$ satisfies the following properties

$$
\begin{gathered}
\frac{1}{2} a_{2 p-1} s^{2 p}-c_{1} \leq f(s) s \leq \frac{3}{2} a_{2 p-1} s^{2 p}+c_{1}, \\
f^{\prime}(s) \geq \frac{1}{2} a_{2 p-1} s^{2 p-2}-c_{2} \geq-k, \forall s \in R, k \geq 0
\end{gathered}
$$

where

$$
F(s)=\int_{0}^{s} f(\tau) \mathrm{d} \tau
$$

such as

$$
\frac{1}{4 p} a_{2 p-1} s^{2 p}-c_{3} \leq F(s) \leq \frac{3}{4 p} a_{2 p-1} s^{2 p}+c_{3}
$$

Remark 2.1. We take here, for simplicity, Dirichlet Boundary Conditions. However, we can obtain the same results for Neumann Boundary Conditions, namely,

$$
\frac{\partial u}{\partial v}=\frac{\partial \Delta u}{\partial v}=\frac{\partial \varphi}{\partial v} \quad \text { on } \Gamma
$$

where $v$ denotes the unit outer normal to $\Gamma$. To do so, we rewrite, owing to (23) and (24), the equations in the form

$$
\begin{gathered}
\frac{\partial \bar{u}}{\partial t}+\Delta^{2} \bar{u}-\Delta(f(u)-\langle f(u)\rangle)=-\Delta \frac{\partial \bar{\alpha}}{\partial t} \\
\frac{\partial^{2} \bar{\varphi}}{\partial t^{2}}+\frac{\partial \bar{\varphi}}{\partial t}-\Delta \bar{\varphi}=-\frac{\partial \bar{u}}{\partial t},
\end{gathered}
$$

where $\bar{v}=v-\langle v\rangle,\left|\left\langle v_{0}\right\rangle\right| \leq M_{1},\left|\left\langle v_{0}\right\rangle\right| \leq M_{2}$, for fixed positive constants $M_{1}$ and $M_{2}$. Then, we note that

$$
v \rightarrow\left(\left\|(-\Delta)^{\frac{-1}{2}} v\right\|^{2}+\langle v\rangle^{2}\right)^{\frac{1}{2}}
$$

where, here, $-\Delta$ denotes the minus Laplace operator with Neumann boundary conditions and acting on functions with null average and where it is understood that

$$
\langle\cdot\rangle=\frac{1}{\operatorname{vol}(\Omega)}\langle\cdot, 1\rangle_{H^{-1}(\Omega), H^{1}(\Omega)}
$$

Furthermore 


$$
\begin{gathered}
v \mapsto\left(\|\bar{v}\|^{2}+\langle v\rangle^{2}\right)^{\frac{1}{2}}, \\
v \mapsto\left(\|\nabla v\|^{2}+\langle v\rangle^{2}\right)^{\frac{1}{2}}, \\
v \mapsto\left(\|\Delta v\|^{2}+\langle v\rangle^{2}\right)^{\frac{1}{2}}
\end{gathered}
$$

are norms in $H^{-1}(\Omega), L^{2}(\Omega), H^{1}(\Omega)$ and $H^{2}(\Omega)$, respectively, which are equivalent to the usual ones.

We further assume that

$$
|f(s)| \leq \varepsilon F(s)+c_{\varepsilon}, \forall \varepsilon>0, \quad s \in R,
$$

which allows to deal with term $\langle f(u)\rangle$.

\section{Notations}

We denote by $\|\cdot\|$ the usual $L^{2}$-norm (with associated product scalar (.,.) and set $\|\cdot\|_{-1}=\left\|(-\Delta)^{\frac{-1}{2}} \cdot\right\|$, where $-\Delta$ denotes the minus Laplace operator with Dirichlet Boundary Conditions. More generally, $\|\cdot\|_{X}$ denote the norm of Banach space $X$.

Throughout this paper, the same letters $c_{1}, c_{2}$ and $c_{3}$ denote (generally positive) constants which may change from line to line, or even a same line.

\section{A Priori Estimates}

The estimates derived in this subsection will be formal, but they can easily be justified within a Galerkin scheme. We rewrite (23) in the equivalent form

$$
(-\Delta)^{-1} \frac{\partial u}{\partial t}-\Delta u+f(u)=\frac{\partial \alpha}{\partial t}
$$

We multiply (35) by $\frac{\partial u}{\partial t}$ and have, integrating over $\Omega$ and by parts;

$$
\frac{\mathrm{d}}{\mathrm{d} t}\left(\|\nabla u\|^{2}+2 \int_{\Omega} F(u) \mathrm{d} x\right)+2\left\|\frac{\partial u}{\partial t}\right\|_{-1}^{2}=2\left(\frac{\partial u}{\partial t}, \frac{\partial \alpha}{\partial t}\right)
$$

We then multiply (24) by $\frac{\partial \alpha}{\partial t}$ to obtain

$$
\frac{\mathrm{d}}{\mathrm{d} t}\left(\|\nabla \alpha\|^{2}+\left\|\frac{\partial \alpha}{\partial t}\right\|^{2}\right)+2\left\|\frac{\partial \alpha}{\partial t}\right\|^{2}=-2\left(\frac{\partial u}{\partial t}, \frac{\partial \alpha}{\partial t}\right)
$$

Summing (36) and (37), we find the differential inequality of the form

$$
\frac{\mathrm{d}}{\mathrm{d} t}\left(\|\nabla u\|^{2}+2 \int_{\Omega} F(u) \mathrm{d} x+\|\nabla \alpha\|^{2}+\left\|\frac{\partial \alpha}{\partial t}\right\|^{2}\right)+2\left\|\frac{\partial u}{\partial t}\right\|_{-1}^{2}+2\left\|\frac{\partial \alpha}{\partial t}\right\|^{2}=0
$$

Integrating from 0 to $t$ with $t \in[0 ; T]$ we obtain 


$$
\begin{aligned}
& \int_{0}^{t}\left(\frac{\mathrm{d}}{\mathrm{d} t}\|\nabla u\|^{2}+2 \int_{\Omega} F(u) \mathrm{d} x+\|\nabla \alpha(s)\|^{2}+\left\|\frac{\partial \alpha(s)}{\partial t}\right\|^{2}\right) \mathrm{d} s \\
& +2 \int\left\|\frac{\partial \alpha(s)}{\partial t}\right\|^{2} \mathrm{~d} s+2 \int\left\|\frac{\partial u(s)}{\partial t}\right\|_{-1}^{2} \mathrm{~d} s=0
\end{aligned}
$$

of (35) we deduce

$$
F\left(u_{0}\right) \leq \frac{3}{4 p} a_{2 p-1} u_{0}^{2 p}+c_{3}
$$

which involves

$$
2 \int_{\Omega} F\left(u_{0}\right) \mathrm{d} x \leq \frac{3}{2 p} a_{2 p-1}\left\|u_{0}\right\|_{L^{2 p}}^{2 p}+2 c_{3}|\Omega|
$$

still of (35) we have

$$
\frac{3}{4 p} a_{2 p-1} u_{0}^{2 p}-c_{3} \leq F(u)
$$

which involves

$$
\frac{1}{2 p} a_{2 p-1}\left\|u_{0}\right\|_{L^{2 p}}^{2 p}-2 c_{3}|\Omega| \leq F(u)
$$

where

$$
E(t)+2 \int_{0}^{t}\left(\left\|\frac{\partial \alpha(s)}{\partial t}\right\|^{2}+\left\|\frac{\partial u(s)}{\partial t}\right\|_{-1}^{2}\right) \mathrm{d} s \leq C
$$

with

$$
E(t)=\|\nabla u(t)\|^{2}+\frac{1}{2 p} a_{2 p-1}\|u(t)\|_{L^{2 p}}^{2 p}+\left\|\frac{\partial \alpha(t)}{\partial t}\right\|^{2}+\|\nabla \alpha(t)\|^{2}
$$

and $C=\left\|\nabla u_{0}\right\|^{2}+\frac{3}{2 p} a_{2 p-1}\left\|u_{0}\right\|_{L^{2 p}}^{2 p}+\left\|\alpha_{1}\right\|^{2}+\left\|\nabla \alpha_{0}\right\|^{2}+C_{3}$.

Finally, we conclude that

$$
\begin{aligned}
& u \in L^{\infty}\left(R^{*} ; H_{0}^{1}(\Omega) \cap L^{2 p}(\Omega)\right) ; \alpha \in L^{2}\left(0, T ; H^{-1}(\Omega)\right) ; \\
& \quad \frac{\partial u}{\partial t} \in L^{2}\left(0, T ; H^{-1}(\Omega)\right) ; \frac{\partial \alpha}{\partial t} \in L^{\infty}\left(R_{+}^{*} ; L^{2}(\Omega)\right) \cap L^{2}\left(0, T ; L^{2}(\Omega)\right) \quad \forall T>0
\end{aligned}
$$

Theorem 4.1. (Existence) We assume $\left(u_{0}, \alpha_{0}, \alpha_{1}\right) \in\left(H_{0}^{1}(\Omega) \cap L^{2 p}(\Omega)\right) \times H_{0}^{1}(\Omega) \times L^{2}(\Omega)$ then the system (18)-(19) possesses at least one solution $(u, \alpha)$ such that

$$
\begin{gathered}
u \in L^{\infty}\left(R^{*} ; H_{0}^{1}(\Omega) \cap L^{2 p}(\Omega)\right) ; \alpha \in L^{2}\left(0, T ; H^{-1}(\Omega)\right) \\
\frac{\partial u}{\partial t} \in L^{2}\left(0, T ; H^{-1}(\Omega)\right) ; \frac{\partial \alpha}{\partial t} \in L^{\infty}\left(R_{+}^{*} ; L^{2}(\Omega)\right) \cap L^{2}\left(0, T ; L^{2}(\Omega)\right) \\
\forall T>0
\end{gathered}
$$

Theorem 4.2. (Uniqueness) Let the assumptions of Theorem 4.1 hold. Then, the system (18)-(19) possesses a unique solution $(u, \alpha)$ such that 


$$
\begin{gathered}
u \in L^{\infty}\left(R^{*} ; H_{0}^{1}(\Omega) \cap L^{2 p}(\Omega)\right) ; \alpha \in L^{2}\left(0, T ; H^{-1}(\Omega)\right) \\
\frac{\partial u}{\partial t} \in L^{2}\left(0, T ; H^{-1}(\Omega)\right) ; \frac{\partial \alpha}{\partial t} \in L^{\infty}\left(R_{+}^{*} ; L^{2}(\Omega) \cap L^{2}\left(0, T ; L^{2}(\Omega)\right)\right. \\
\forall T>0
\end{gathered}
$$

Let $\left(u^{(1)}, \alpha^{(1)}, \frac{\partial \alpha^{(1)}}{\partial t}\right)$ and $\left(u^{(2)}, \alpha^{(2)}, \frac{\partial \alpha^{(2)}}{\partial t}\right)$ be two solutions (23)-(25) with initial data $\left(u_{0}^{(1)}, \alpha_{0}^{(1)}, \alpha_{1}^{(1)}\right)$ and $\left(u_{0}^{(2)}, \alpha_{0}^{(2)}, \alpha_{1}^{(2)}\right)$, respectively. We set

$$
\left(u, \alpha, \frac{\partial \alpha}{\partial t}\right)=\left(u^{(1)}, \alpha^{(1)}, \frac{\partial \alpha^{(1)}}{\partial t}\right)-\left(u^{(2)}, \alpha^{(2)}, \frac{\partial \alpha^{(2)}}{\partial t}\right)
$$

and

$$
\left(u_{0}, \alpha_{0}, \alpha_{1}\right)=\left(u_{0}^{(1)}, \alpha_{0}^{(1)}, \alpha_{1}^{(1)}\right)-\left(u_{0}^{(2)}, \alpha_{0}^{(2)}, \alpha_{1}^{(2)}\right)
$$

Then, $(u, \alpha)$ satisfies

$$
\begin{gathered}
\frac{\partial u}{\partial t}+\Delta^{2} u-\Delta\left(f\left(u^{(1)}\right)-f\left(u^{(2)}\right)\right)=-\Delta \frac{\partial \alpha}{\partial t} \\
\frac{\partial^{2} \alpha}{\partial t^{2}}+\frac{\partial \alpha}{\partial t}-\Delta \alpha=-\frac{\partial u}{\partial t} \\
\left.u\right|_{\Gamma}=\left.\Delta u\right|_{\Gamma}=\left.\alpha\right|_{\Gamma}=0, \quad \text { on } \partial \Omega, \\
\left.u\right|_{t=0}=u_{0},\left.\alpha\right|_{t=0}=\alpha_{0}, \frac{\partial \alpha}{\partial t}=\alpha_{1}
\end{gathered}
$$

We multiply (40) by $(-\Delta)^{-1} \frac{\partial u}{\partial t}$, we have

$$
\begin{gathered}
\left\|\frac{\partial u}{\partial t}\right\|_{-1}^{2}+\left(\frac{\partial u}{\partial t},-\Delta u\right)+\left(-\Delta\left(f\left(u^{(1)}\right)-f\left(u^{(2)}\right)\right),(-\Delta)^{-1} \frac{\partial u}{\partial t}\right)=\left(\frac{\partial u}{\partial t}, \frac{\partial \alpha}{\partial t}\right) \\
\frac{\mathrm{d}}{\mathrm{d} t}\|\nabla u\|^{2}+2\left\|\frac{\partial u}{\partial t}\right\|_{-1}^{2}=-2\left(f\left(u^{(1)}\right)-f\left(u^{(2)}\right), \frac{\partial u}{\partial t}\right)+2\left(\frac{\partial u}{\partial t}, \frac{\partial \alpha}{\partial t}\right) .
\end{gathered}
$$

We multiply by (41) by $\frac{\partial \alpha}{\partial t}$, we have

$$
\frac{\mathrm{d}}{\mathrm{d} t}\left(\|\nabla \alpha\|^{2}+\left\|\frac{\partial \alpha}{\partial t}\right\|^{2}\right)+2\left\|\frac{\partial \alpha}{\partial t}\right\|^{2}=-2\left(\frac{\partial u}{\partial t}, \frac{\partial \alpha}{\partial t}\right)
$$

Now summing (44) and (45) we obtain

$$
\begin{aligned}
& \frac{\mathrm{d}}{\mathrm{d} t}\left(\|\nabla u\|^{2}+\|\nabla \alpha\|^{2}+\left\|\frac{\partial \alpha}{\partial t}\right\|^{2}\right)+2\left\|\frac{\partial u}{\partial t}\right\|_{-1}^{2}+2\left\|\frac{\partial \alpha}{\partial t}\right\|^{2} \\
& =-2\left(f\left(u^{(1)}\right)-f\left(u^{(2)}\right), \frac{\partial u}{\partial t}\right)
\end{aligned}
$$

We know that

$$
f\left(u^{1}\right)-f\left(u^{2}\right)=\sum_{k=1}^{2 p-1} a_{k}\left(u^{(1) k}\right)-\sum_{k=1}^{2 p-1} a_{k}\left(u^{(2) k}\right)=\sum_{k=1}^{2 p-1} a_{k}\left(u^{(1) k}-u^{(2) k}\right)
$$


which involves

$$
\begin{aligned}
& \left|f\left(u^{1}\right)-f\left(u^{2}\right)\right| \leq \sum_{k=1}^{2 p-1}\left|a_{k}\right|\left|u^{(1) k}-u^{(2) k}\right| \\
& \leq \sum_{k=1}^{2 p-1}\left|a_{k}\right|\left|u^{(1)}-u^{(2)}\right|\left|u^{(1)}\right|^{k-1}+\sum_{j=1}^{k-2}\left|u^{(1)}\right|^{k-1-j}\left|u^{(2)}\right|^{j}+\left|u^{(2)}\right|^{k-1} .
\end{aligned}
$$

Based on Young's inequality, we have

$$
\sum_{j=1}^{k-2}\left|u^{(1)}\right|^{k-1-j}\left|u^{(2)}\right|^{j} \leq \sum_{j=1}^{k-2}\left(\frac{k-j-1}{k-1}\left|u^{(1)}\right|^{k-1}+\frac{j}{k-1}\left|u^{(2)}\right|^{k-1}\right)
$$

with $p=\frac{k-1}{k-j-1}$ and $q=\frac{k-1}{j}$ such as $\frac{1}{p}+\frac{1}{q}=1$. So

$$
\sum_{j=1}^{k-2}\left|u^{(1)}\right|^{k-1-j}\left|u^{(2)}\right|^{j} \leq \frac{1}{k-1} \sum_{j=1}^{k-2}(k-1)\left|u^{(1)}\right|^{k-1}+\frac{1}{k-1} \sum_{j=1}^{k-2} j\left(\left|u^{(2)}\right|^{k-1}-\left|u^{(1)}\right|^{k-1}\right) \text {. }
$$

As

$$
\sum_{j=1}^{k-2} j=\frac{(k-2)(k-1)}{2}
$$

then

$$
\begin{aligned}
\sum_{j=1}^{k-2}\left|u^{(1)}\right|^{k-1-j}\left|u^{(2)}\right|^{j} & \leq(k-2)\left|u^{(1)}\right|^{k-1}+\frac{k-2}{2}\left|u^{(2)}\right|^{k-1}-\frac{k-2}{2}\left|u^{(1)}\right|^{k-1} \\
& \leq \frac{k-2}{2}\left(\left|u^{(1)}\right|^{k-1}+\left|u^{(2)}\right|^{k-1}\right) .
\end{aligned}
$$

We know that

$$
\begin{aligned}
& \forall k \in N ; \quad k-2 \leq k \text { then } \frac{k-2}{2} \leq \frac{k}{2} \leq k \\
& \sum_{j=1}^{k-2}\left|u^{(1)}\right|^{k-1-j}\left|u^{(2)}\right|^{j} \leq k\left(\left|u^{(1)}\right|^{k-1}+\left|u^{(2)}\right|^{k-1}\right)
\end{aligned}
$$

which gives

$$
\begin{aligned}
\left|f\left(u^{1}\right)-f\left(u^{2}\right)\right| & \leq \sum_{j=1}^{k-2}\left|a_{k}\right|\left|u^{(1)}-u^{(2)}\right|\left((k+1)\left|u^{(1)}\right|^{k-1}+(k+1)\left|u^{(2)}\right|^{k-2}\right) \\
& \leq|u| \sum_{j=1}^{k-2}(k+1)\left|a_{k}\right|\left(\left|u^{(1)}\right|^{k-1}+\left|u^{(2)}\right|^{k-1}\right)
\end{aligned}
$$

$\exists k>0$ such as

$$
(k+1)\left|a_{k}\right| \leq k ; \quad \forall k \in 1,2, \cdots, 2 p-1
$$

so

$$
\left|f\left(u^{1}\right)-f\left(u^{2}\right)\right| \leq|u| k \sum_{k=1}^{k-2}\left(\left|u^{(1)}\right|^{k-1}+\left|u^{(2)}\right|^{k-1}\right) .
$$

Based on Young's inequality, we have $\forall k \geq 2$

$$
\left|u^{(1)}\right|^{k-1} \leq \frac{k-1}{2 p-2}\left(\left|u^{(1)}\right|^{k-1}\right)^{\frac{2 p-2}{k-1}}+\frac{2 p-k-1}{2 p-2}
$$


and

$$
\left|u^{(2)}\right|^{k-1} \leq \frac{k-1}{2 p-2}\left(\left|u^{(2)}\right|^{k-1}\right)^{\frac{2 p-2}{k-1}}+\frac{2 p-k-1}{2 p-2}
$$

that involve

$$
\begin{aligned}
\left|f\left(u^{1}\right)-f\left(u^{2}\right)\right| & \leq|u| \frac{k}{2 p-2} \sum_{k=1}^{2 p-1}\left((k-1)\left(\left|u^{(1)}\right|^{2 p-2}+\left|u^{(2)}\right|^{2 p-2}\right)+2\left(\frac{2 p-k-1}{2 p-2}\right)\right) \\
& \leq c|u|\left(\left|u^{(1)}\right|^{2 p-2}+\left|u^{(2)}\right|^{2 p-2}+1\right) .
\end{aligned}
$$

We finally

$$
\int_{\Omega}\left|f\left(u^{1}\right)-f\left(u^{2}\right)\right|\left|\frac{\partial u}{\partial t}\right| \mathrm{d} x \leq c \int_{\Omega}|u|\left(\left|u^{(1)}\right|^{2 p-2}+\left|u^{(2)}\right|^{2 p-2}+1\right)\left|\frac{\partial u}{\partial t}\right| \mathrm{d} x .
$$

The second member of (45) is increased in $R^{n}$ for $n=1,2,3$.

If $n=1 ; u^{i} \in H_{0}^{1}(\Omega) \subset H^{1}(\Omega)=W^{1,2}(\Omega)$ for $i=1,2$.

Thanks to the continuous injection $H^{1}(\Omega) \subset C(\bar{\Omega})$, then is $C>0$, by applying Holder's inegality, we get

$$
\int_{\Omega}|u|\left(\left|u^{(1)}\right|^{2 p-2}+\left|u^{(1)}\right|^{2 p-2}+1\right)\left|\frac{\partial u}{\partial t}\right| \mathrm{d} x \leq C\|u\|\left\|\frac{\partial u}{\partial t}\right\|,
$$

which involves using the compact injection $H^{1}(\Omega) \subset L^{2}(\Omega)$, we have

$$
\int_{\Omega}\left|f\left(u^{1}\right)-f\left(u^{2}\right)\right| \frac{\partial u}{\partial t} \mid \mathrm{d} x \leq C\|u\|_{H^{1}}\left\|\frac{\partial u}{\partial t}\right\|
$$

If $n=2$ then $H^{1}(\Omega) \subset L^{q}(\Omega), \forall q \in[1, \infty[$.

Based on Holder's inequality, we have

$$
\int_{\Omega}|u|\left(\left|u^{(1)}\right|^{2 p-2}+\left|u^{(1)}\right|^{2 p-2}+1\right)\left|\frac{\partial u}{\partial t}\right| \mathrm{d} x \leq C\|u\|_{L^{3}}\left\|\frac{\partial u}{\partial t}\right\| .
$$

Finally

$$
\int_{\Omega}\left|f\left(u^{1}\right)-f\left(u^{2}\right)\right|\left|\frac{\partial u}{\partial t}\right| \mathrm{d} x \leq C\|u\|_{H^{1}}\left\|\frac{\partial u}{\partial t}\right\|
$$

If $n=3$, then $H^{1}(\Omega) \subset L^{q}(\Omega)$ with $q \in[1,6]$

In this case, we also

$$
\int_{\Omega}|u|\left(\left|u^{(1)}\right|^{2 p-2}+\left|u^{(1)}\right|^{2 p-2}+1\right)\left|\frac{\partial u}{\partial t}\right| \mathrm{d} x \leq C\|u\|_{L^{6}}\left\|\frac{\partial u}{\partial t}\right\| .
$$

So

$$
\int_{\Omega}\left|f\left(u^{1}\right)-f\left(u^{2}\right)\right|\left|\frac{\partial u}{\partial t}\right| \mathrm{d} x \leq C\|u\|_{H^{1}} \| \frac{\partial u}{\partial t} \mid .
$$

We notice that in $R^{n}$ for $n=1,2,3$, we have

$$
\int_{\Omega}\left|f\left(u^{1}\right)-f\left(u^{2}\right)\right|\left|\frac{\partial u}{\partial t}\right| \mathrm{d} x \leq C\|u\|_{H^{1}} \| \frac{\partial u}{\partial t} \mid .
$$

Using Young's inequality, we have 


$$
\int_{\Omega}\left|f\left(u^{1}\right)-f\left(u^{2}\right)\right| \frac{\partial u}{\partial t} \mid \mathrm{d} x \leq C\|u\|_{H^{1}}^{2}+\left\|\frac{\partial u}{\partial t}\right\|^{2}
$$

Inserting (49) into (46), we find

$$
\frac{\mathrm{d}}{\mathrm{d} t} E_{2}+2\left\|\frac{\partial u}{\partial t}\right\|_{-1}^{2}+2\left\|\frac{\partial \alpha}{\partial t}\right\|^{2} \leq c^{\prime}\|u\|_{H^{1}}^{2}+\left\|\frac{\partial u}{\partial t}\right\|^{2}
$$

and recalling the interpolation inequality $\left\|\frac{\partial u}{\partial t}\right\|^{2} \leq c\left\|\frac{\partial u}{\partial t}\right\|_{-1}\left\|\nabla \frac{\partial u}{\partial t}\right\|$

with $E_{2}=\|\nabla u\|^{2}+\|\nabla \alpha\|^{2}+\left\|\frac{\partial \alpha}{\partial t}\right\|^{2}$

Finally

$$
\frac{\mathrm{d}}{\mathrm{d} t} E_{2}+c^{\prime \prime}\left\|\frac{\partial u}{\partial t}\right\|_{-1}^{2}+2\left\|\frac{\partial \alpha}{\partial t}\right\|^{2} \leq C E_{2}, \quad C>0
$$

Theorem 4.3. (Second theorem of the solution's existence) The existence and uniqueness of the solution (23)-(25) problem being proven, now we seek the solution of (23)-(25) with more regularity.

$$
\text { Assume } \begin{aligned}
& \left(u_{0}, \alpha_{0}, \alpha_{1}\right) \in H^{2}(\Omega) \cap H_{0}^{1}(\Omega) \cap L^{2 p}(\Omega) \\
& \times\left(u_{0}, \alpha_{0}, \alpha_{1}\right) \in H^{2}(\Omega) \cap H_{0}^{1}(\Omega) \cap L^{2 p}(\Omega) \times H_{0}^{1}(\Omega)
\end{aligned} \text {, then the }
$$

(23)-(24) system admits a unique $(u, \alpha)$ solution such as

$$
\begin{aligned}
& u \in L^{\infty}\left(0, T ; H^{2}(\Omega) \cap H_{0}^{1}(\Omega)\right), \alpha \in L^{\infty}\left(0, T ; H^{2}(\Omega) \cap H_{0}^{1}(\Omega)\right), \\
& \frac{\partial \alpha}{\partial t} \in L^{\infty}\left(0, T ; H^{2}(\Omega) \cap H_{0}^{1}(\Omega)\right) \cap L^{2}\left(0, T ; H^{2}(\Omega) \cap H_{0}^{1}(\Omega)\right),
\end{aligned}
$$

and

$$
\frac{\partial u}{\partial t} \in L^{2}\left(0, T ; H^{-1}(\Omega)\right)
$$

Theorems of existence (23) and uniqueness (24) being proven then $u \in L^{\infty}\left(0, T ; H^{2}(\Omega) \cap L^{2 p}(\Omega)\right), \quad \alpha \in L^{\infty}\left(0, T ; H_{0}^{1}(\Omega)\right)$, $\frac{\partial \alpha}{\partial t} \in L^{\infty}\left(0, T ; L^{2}(\Omega)\right) \cap L^{2}\left(0, T ; L^{2}(\Omega)\right)$ and $\frac{\partial u}{\partial t} \in L^{\infty}\left(0, T ; H^{-1}(\Omega)\right), \quad \forall T>0$.

We multiply (23) by $(-\Delta)^{-1} \frac{\partial u}{\partial t}$ and have, integrating over $\Omega$, we have

$$
\frac{\mathrm{d}}{\mathrm{d} t}\left(\|\nabla u\|^{2}+2 \int_{\Omega} F(u) \mathrm{d} x\right)+2\left\|\frac{\partial u}{\partial t}\right\|_{-1}^{2}=2\left(\frac{\partial u}{\partial t}, \frac{\partial \alpha}{\partial t}\right)
$$

Multiplying (24) by $\frac{\partial \alpha}{\partial t}$, we have

$$
\frac{\mathrm{d}}{\mathrm{d} t}\left(\|\nabla \alpha\|^{2}+\left\|\frac{\partial \alpha}{\partial t}\right\|^{2}\right)+2\left\|\frac{\partial \alpha}{\partial t}\right\|^{2}=-2\left(\frac{\partial u}{\partial t}, \frac{\partial \alpha}{\partial t}\right)
$$

Now summing (51) and (52) we obtain

$$
\frac{\mathrm{d}}{\mathrm{d} t}\left(\|\nabla u\|^{2}+2 \int_{\Omega} F(u) \mathrm{d} x+\|\nabla \alpha\|^{2}+\left\|\frac{\partial \alpha}{\partial t}\right\|^{2}\right)+2\left\|\frac{\partial u}{\partial t}\right\|_{-1}^{2}+2\left\|\frac{\partial \alpha}{\partial t}\right\|^{2}=0
$$


where

$$
E_{3}=\|\nabla u\|^{2}+2 \int_{\Omega} F(u) \mathrm{d} x+\|\nabla \alpha\|^{2}+\left\|\frac{\partial \alpha}{\partial t}\right\|^{2}
$$

finally

$$
\|\nabla u(t)\|^{2}+c\|u(t)\|_{L^{2}}^{2 p}+\|\nabla \alpha(t)\|^{2}+\left\|\frac{\partial \alpha}{\partial t}\right\|^{2}+2 \int_{0}^{t}\left(\left\|\frac{\partial \alpha(s)}{\partial t}\right\|^{2}+\left\|\frac{\partial u(s)}{\partial t}\right\|_{-1}^{2}\right) \mathrm{d} s \leq c_{1} .
$$

We infer that

$$
\begin{gathered}
u \in L^{\infty}\left(0, T ; H^{2}(\Omega) \cap L^{2 p}(\Omega)\right), \quad \alpha \in L^{\infty}\left(0, T ; H_{0}^{1}(\Omega)\right), \\
\frac{\partial \alpha}{\partial t} \in L^{\infty}\left(0, T ; L^{2}(\Omega)\right) \cap L^{2}\left(0, T ; L^{2}(\Omega)\right) \text { and } \frac{\partial u}{\partial t} \in L^{\infty}\left(0, T ; H^{-1}(\Omega)\right) .
\end{gathered}
$$

We multiply (24) by $\frac{\partial^{2} \alpha}{\partial t^{2}}$, we have

$$
\frac{\mathrm{d}}{\mathrm{d} t}\left(\left\|\frac{\partial \alpha}{\partial t}\right\|^{2}+\|\nabla \alpha\|^{2}\right)+\left\|\frac{\partial^{2} \alpha}{\partial t^{2}}\right\|^{2} \leq\left\|\frac{\partial u}{\partial t}\right\|^{2} .
$$

We infer from this that $\frac{\partial^{2} \alpha}{\partial t^{2}} \in L^{2}\left(0, T ; L^{2}(\Omega)\right)$.

\section{Conclusion}

In this work we have studied the existence and uniqueness of the solution of a conservative-type Caginalp system with Dirichlet-type boundary conditions. Finally we have also succeeded in this work to establish the existence theorems of the solution of this system with low regularity and more regularity. As a perspective, we plan to study this problem in a bounded or unbounded domain with different types of potentials and Neumann-type conditions.

\section{Conflicts of Interest}

The authors declare no conflicts of interest regarding the publication of this paper.

\section{References}

[1] Altundas, Y.B. and Caginalp, G. (2005) Velocity Selection in 3D Dendrites: Phase Field Computations and Microgravity Experiments. Nonlinear Analysis, 62, 467-481. https://doi.org/10.1016/j.na.2005.02.122

[2] Babin, A.V. and Vishik, M.I. (1992) Attractors of Evolution Equations. Vol. 25 of Studies in Mathematics and Its Applications, North-Holland Publishing Co., Amsterdam.

[3] Batangouna, N. and Pierre, M. (2018) Convergence of Exponential Attractors for a Time Splitting Approximation of the Caginalp Phase-Field System. Communications on Pure \& Applied Analysis, 17, 1-19. https://doi.org/10.3934/cpaa.2018001

[4] Bai, F., Elliott, C.M., Gardiner, A., et al. (1995) The Viscous Cahn-Hilliard Equation. I. Computations. Nonlinearity, 8, 131-160. https://doi.org/10.1088/0951-7715/8/2/002

[5] Bates, P.W. and Zheng, S.M. (1992) Inertial Manifolds and Inertial Sets for the 
Phase-Field Equations. Journal of Dynamics and Differential Equations, 4, 375-398. https://doi.org/10.1007/BF01049391

[6] Brezis, H. (1973) Opérateurs maximaux monotones et semi-groupes de contractions dans les espaces de Hilbert. North-Holland Publishing Co., Amsterdam, London, American Elsevier Publishing Co. Inc., New York.

[7] Brochet, D., Hilhorst, D. and Chen, X. (1993) Finite-Dimensional Exponential Attractor for the Phase Field Model. Applicable Analysis, 49, 197-212. https://doi.org/10.1080/00036819108840173

[8] Brokate, M. and Sprekels, J. (1996) Hysteresis and Phase Transitions. Vol. 121 of Applied Mathematical Sciences, Springer-Verlag, New York. https://doi.org/10.1007/978-1-4612-4048-8

[9] Landau, L.D. and Lifshitz, E.M. (1980) Statistical Physics I. 3rd Edition, Butterworth-Heinemann, Oxford.

[10] Caginalp, G. and Socolovsky, E.A. (1989 Efficient Computation of a Sharp Interface by Spreading via Phase Field Methods. Applied Mathematics Letters, 2, 117-120. https://doi.org/10.1016/0893-9659(89)90002-5

[11] Caginalp, G. (1986) An Analysis of a Phase Field Model of a Free Boundary. Archive for Rational Mechanics and Analysis, 92, 205-245. https://doi.org/10.1007/BF00254827

[12] Chen, P.J., Gurtin, M.E. and Williams, W.O. (1968) A Note on Non-Simple Heat Conduction. Journal of Applied Physics (ZAMP), 19, 969-970. https://doi.org/10.1007/BF01602278

[13] Chepyzhov, V.V. and Vishik, M.I. (2002) Attractors for Equations of Mathematical Physics. Vol. 49 of American Mathematical Society Colloquium Publications, American Mathematical Society, Providence. https://doi.org/10.1090/coll/049

[14] Chill, R., Fašangová, E. and Prüss, J. (2006) Convergence to Steady State of Solutions of the Cahn-Hilliard and Caginalp Equations with Dynamic Boundary Conditions. Mathematische Nachrichten, 279, 1448-1462. https://doi.org/10.1002/mana.200410431

[15] Dupaix, C., Hilhorst, D. and Kostin, I.N. (1999) The Viscous Cahn-Hilliard Equation as a Limit of the Phase Field Model: Lower Semicontinuity of the Attractor. Journal of Dynamics and Differential Equations, 11, 333-353. https://doi.org/10.1023/A:1021985631123

[16] Doumbe, B. (2013) Etude de modeles de champ de phase de type Caginalp. PhD Thesis, Université de Poiters.

[17] Efendiev, M., Miranville, A. and Zelik, S. (2000) Exponential Attractors for a Nonlinear Reaction-Diffusion System in $R^{3}$. Comptes Rendus de P Académie des Sciences-Series I-Mathematics, 330, 713-718. https://doi.org/10.1016/S0764-4442(00)00259-7

[18] Efendiev, M., Miranville, A. and Zelik, S. (2004) Exponential Attractors for a Singularly Perturbed Cahn-Hilliard System. Mathematische Nachrichten, 272, 11-31. https://doi.org/10.1002/mana.200310186

[19] Elliott, C.M. and Stuart, A.M. (1993) The Global Dynamics of Discrete Semilinear Parabolic Equations. SIAM Journal on Numerical Analysis, 30, 1622-1663. https://doi.org/10.1137/0730084

[20] Miranville, A. and Quintanilla, R. (2009) Some Generalizations of the Caginalp Phase-Field System. Applicable Analysis, 88, 877-894. https://doi.org/10.1080/00036810903042182

[21] Miranville, A. (2014) Some Mathematical Models in Phase Transition. Discrete \& 
Continuous Dynamical Systems Series S, 7, 271-306.

https://doi.org/10.3934/dcdss.2014.7.271

[22] Miranville, A. and Quintanilla, R. (2016) A Caginalp Phase-Field System Based on Type III Heat Conduction with Two Temperatures. Quarterly of Applied Mathematics, 74, 375-398. https://doi.org/10.1090/qam/1430

[23] Penrose, O. and Fife, P.C. (1990) Thermodynamically Consistent Models of PhaseField Type for the Kinetics of Phase Transitions. Journal of Physics D, 43, 44-62. https://doi.org/10.1016/0167-2789(90)90015-H

[24] Quintanilla, R. (2009) A Well-Posed Problem for the Three-Dual-Phase-Lag Heat Conduction. Journal of Thermal Stresses, 32, 1270-1278.

https://doi.org/10.1080/01495730903310599

[25] Temam, R. (1997) Infinite-Dimensional Dynamical Systems in Mechanics and Physics. Vol. 68 of Applied Mathematical Sciences, 2nd Edition, Springer-Verlag, New York. https://doi.org/10.1007/978-1-4612-0645-3

[26] Mavoungou, U.C. (2016) Existence and Uniqueness of Solution for Caginalp Hyperbolic Phase-Field System with a Singular Potential.

[27] Fakih, H. (2015) A Cahn Hilliard Equation with a Proliferation Term for Biological and Chemical Applications. Asymptotic Analysis, 94, 71-104. https://doi.org/10.3233/ASY-151306

[28] Ntsokongo, A.J. and Batangouna, N. (2016) Existence and Uniqueness of Solutions for a Conserved Phase-Field Type Model. AIMS Mathematics, 1, 144-155.

https://doi.org/10.3934/Math.2016.2.144

[29] Raugel, G. (2002) Global Attractors in Partial Differential Equations. In: Handbook of Dynamical Systems, Vol. 2, North-Holland, Amsterdam, 885-982. https://doi.org/10.1016/S1874-575X(02)80038-8

[30] Stuart, A.M. and Humphries, A.R. (1996) Dynamical Systems and Numerical Analysis. Vol. 2 of Cambridge Monographs on Applied and Computational Mathematics, Cambridge University Press, Cambridge.

[31] Temam, R. (1969) Sur l'approximation de la solution des équations de Navier-Stokes par la méthode des pas fractionnaires. II. Archive for Rational Mechanics and Analysis, 33, 377-385. https://doi.org/10.1007/BF00247696

[32] Zhang, Z. (2005) Asymptotic Behavior of Solutions to the Phase-Field Equations with Neumann Boundary Conditions. Communications on Pure \& Applied Analysis, 4, 683-693. https://doi.org/10.3934/cpaa.2005.4.683

[33] Zhu, C. (2015) Attractor of a Semi-Discrete Benjamin-Bona-Mahony Equation on $\mathrm{R}^{1}$. Annales Polonici Mathematici, 115, 219-234. https://doi.org/10.4064/ap115-3-2 